\title{
METASTATIC RENAL CELL CARCINOMA PRESENTING WITH EPISTAXIS
}

\author{
D R Nayak,* K Pujary**, S Ramnani***, C Shetty**, P Parul**
}

\begin{abstract}
Renal cell carcinoma is a slow growing malignancy of the kidney that has a high propensity for metastasis. It is the most common metastasis reported in the paranasal sinuses. A fifty six year old male patient, an operated case of left renal cell carcinoma presented seven years later with epistaxis. Computerized tomography of the paranasal sinuses and of the abdomen showed a metastatic lesion involving the left nasosinus region and a lesion involving the right kidney with lung metastasis, respectively. Due to the poor prognosis palliative radiotherapy was given.
\end{abstract}

Keywords : Renal cell carcinoma; Metastatic; Paranasal sinus

\section{INTRODUCTION}

Grawitz tumor or renal cell carcinoma is a term given for adenocarcinoma of the kidney which more commonly affects males in the 30-60 age group ${ }^{1}$. It is a slow growing tumor and often noticed after metastasis oecurs. The lungs, liver and bone are common sites for metastasis ${ }^{2}$.In the head and neck region, the nose and the paranasal sinuses is the commonest site for metastasis ${ }^{2}$. The presentation is usually with profuse epistaxis and has to be differentiated from vascular lesions, granulomas and primary tumors. To prove the tumor by histopathology may sometimes be difficult due to intractable bleeding and associated necrosis ${ }^{2}$.

\section{CASE REPORT}

A fifty six year old male patient was referred to us with history of left sided epistaxis, facial pain and headache of ten days duration. Seven years prior to this, the patient had undergone left side radical nephrectomy for renal cell carcinoma.

The patient had a postnasal pack which was removed. A diagnostic nasal endoscopy showed clots which was removed revealing a polyp from the middle meatus with an adjacent friable mass. A pulsatile mass was seen in the left sphenoethmoid recess. A CT Scan with contrast done showed an intensely enhancing soft tissue mass involving and expanding the sphenoid sinus with destruction of the roof and extension of the soft tissue lesion into the sella. The pituitary was not visualized separately from the lesion. The mass was extending laterally into the cavernous sinus with compression of the left internal carotid artery. Anteriorly, the lesion was involving the left ethmoids with destruction of the lamina papyracea and minimal extension into the left orbit. Inferiorly the extension was into the left nasal cavity with displacement of the nasal septum to the right and destruction of the medial wall of the maxillary sinus above the level of the inferior turbinate. A CT scan of the thorax and abdomen with contrast was done. It showed a heterogeneously enhancing soft tissue density mass with central areas of necrosis noted superomedial to the right kidney, in the region of the right adrenal gland. Focal loss of fat planes was noted between the mass in the region of the upper pole of the right kidney and segment VII of the right lobe of the liver, suggestive of infiltration. The left kidney was not visualized. Multiple rounded soft tissue density lesions in the basal segments of the right lower lobe of lung was noted. Small nodular opacity in the right middle lobe and another in the left lung lower lobe was noted. These were suggestive of lung metastasis.

Due to profuse recurrent bleeding biopsy could not be taken and surgicel was placed at the site of bleed with a overlying BIPP anterior nasal pack. The poor prognosis was explained to the patient who opted for palliative radiotherapy at another hospital close to his residence.

\section{DISCUSSION}

Renal cell carcinoma is the third most common infraclavicular tumour to metastasize to the head and neck ${ }^{3}$. The incidence of renal cell carcinoma metastasizing to the head and neck has been reported to range from $15-30 \%$. Kostner found 36 cases involving the paranasal sinuses out of 51 cases of metastatic renal cell carcinoma of the head and neck ${ }^{4}$. Other sites of metastasis in the head and neck being the larynx, the jaws, the temporal bones, the ethmoids and the parotid gland. In the nose and paranasal sinuses $^{3,5}$, the maxillary sinus $(36 \%)$ followed by the ethmoid sinus $(25 \%)$, frontal sinus $(17 \%)$ and nasal cavity $(11 \%)$ are affected ${ }^{6}$. Rarely, there may be bilateral maxillary sinus involvement $^{2}$.

The metastatic spread of the tumour is mainly via a haematogenous route. Various haematogenous routes for spread have been described: 1) invasion of the local vascular network by direct extension into the interlobular, arcuate and interlobular veins ${ }^{5}$; 2) through an atrial septal defect $^{7}$; 3) bypass of the pulmonary capillary filtration mechanism via Batson's plexus of anastomosis between avalvular prevertebral, vertebral and epidural venous systems ${ }^{8}$; 4) tumour embolus travels past the IVC to an intercostal or azygous vein in an anterograde manner and move through the same vessel in a retrograde fashion to reach the vertebral venous plexus ${ }^{7}$; 5) associated multiple

*Professor, "Associate Professor, *** Resident, Department of Otolaryngology - Head \& Neck Surgery, Department of Radiology, Kasturba Medical College, Manipal 576104, India

Indian Journal of Otolaryngology and Head and Neck Surgery Vol. 58, No. 4, October-December 2006- 


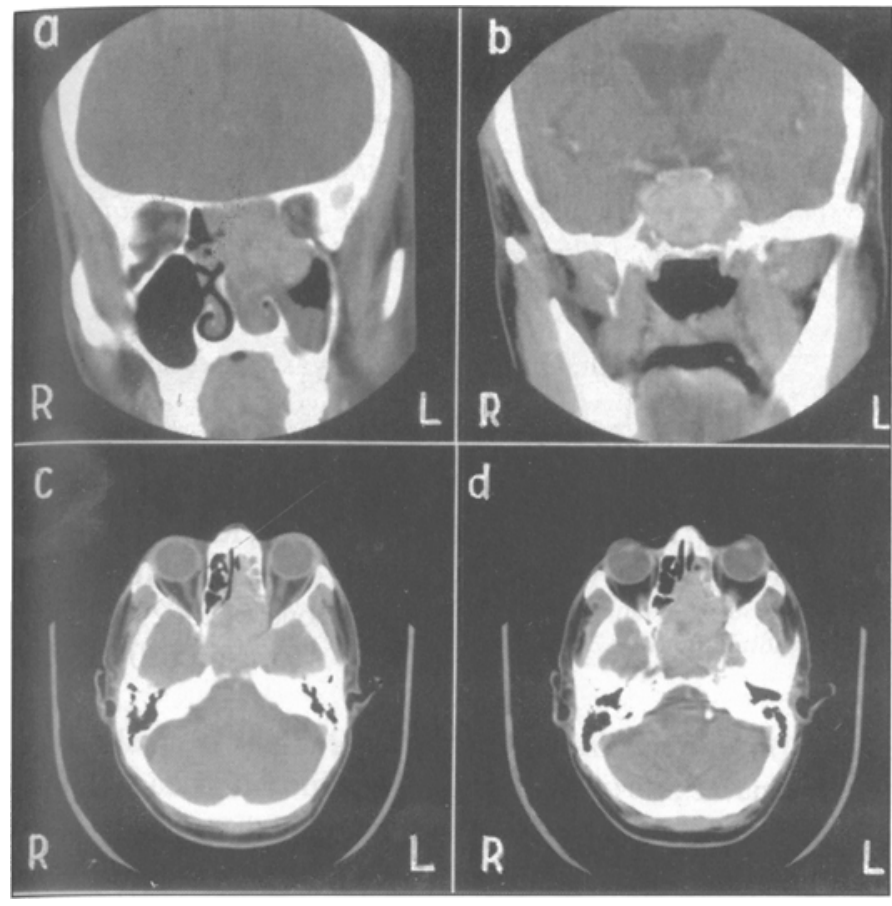

Fig $I I$ : CT scan paranasal sinus (coronal and axial cuts) showing an enhancing lesion involving the left nasosinus region with the extension into the left orbit and erosion of the sphenoid sinus.

arteriovenous shunts ${ }^{9}$. An alternative lymphatic route via regional lymphatics into the thoracic duct could reach the head and neck via retrograde flow through intercostal, mediastinal or supraclavicular lymph vessels ${ }^{7}$. Microscopic seeding of the lung, parenchyma may regress by an immunologic mechanism ${ }^{10}$.

Epistaxis is the common presentation of a sinonasal metastatic renal cell carcinoma and may be the presenting symptom prior to detection of the primary ${ }^{2}$.The epistaxis is often profuse with frequent episodes. The other symptoms are nasal obstruction, facial swelling, pain and headache ${ }^{2}$.

The clinical course of the primary tumour is often unpredictable ${ }^{11}$. Spontaneous regression and also late metastasis after resection of the primary tumour has been noted ${ }^{10,11}$. Most cases of metastatic renal cell carcinoma have a poor prognosis. The prognosis of a solitary metastasis after treatment of the primary is better than those presenting with concurrent metastasis ${ }^{12}$. The treatment of the metastatic lesion is controversial. Surgical removal may prolong the survival of the patient ${ }^{2}$. Though renal cell carcinoma is said to be radioresistant, the metastatic lesion responds well to radiotherapy ${ }^{13}$. Survival of patients treated with surgery is not significantly greater than with full dose radiotherapy. Immunotherapy especially with interleukin-2 and has shown to produce lasting remissions ${ }^{12,15}$. Chemotherapy has not been a successful alternative with poor responses ${ }^{12}$. In our case, the patient had a poor prognosis due to extensive involvement of the other kidney, multiple lung metastasis, and the metastatic lesion in the nose and paranasal sinus which was extending intracranially and involving the cavernous sinus. Palliative radiotherapy was suggested to reduce the tumour bulk and also to reduce the possibility of recurrent epistaxis.
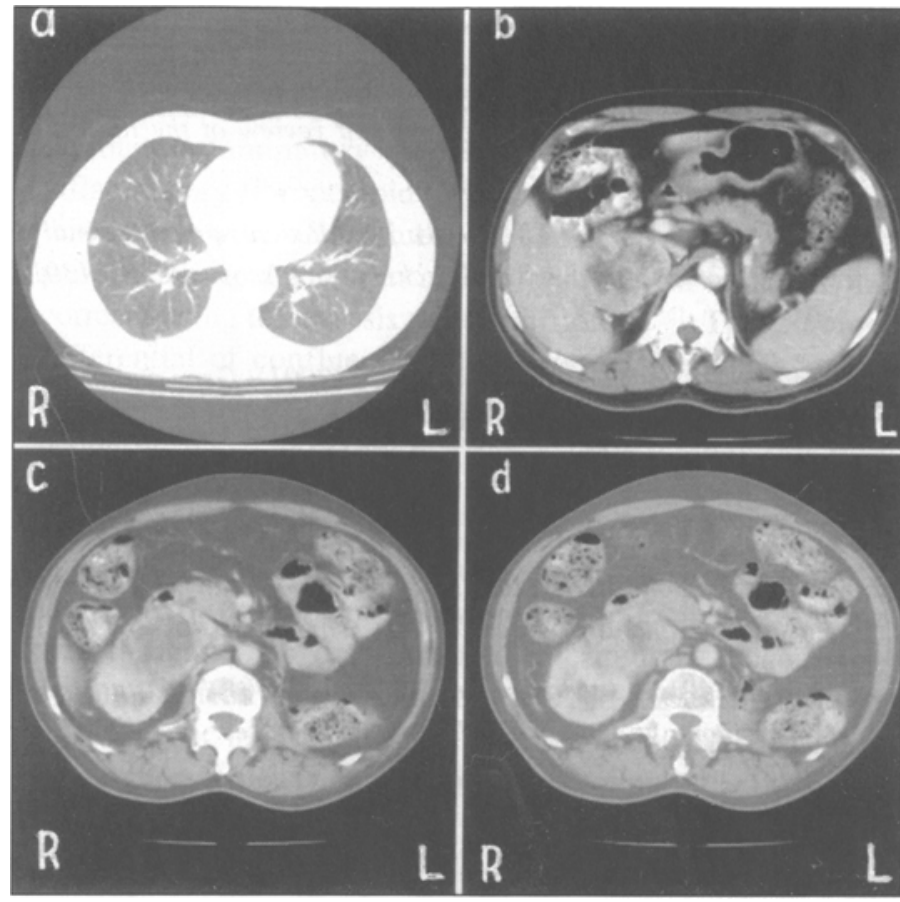

Fig II: CT abdomen and thorax showing a) metastatic lesion in the lower left lobe of the lung; b) lesion infiltrating the liver; c) \& d) enhancing lesion in superomedial aspect of right kidney and absence of left kidney.

In a sinonasal lesion with epistaxis, other than primary malignancies of the paranasal sinuses, a haemangioma, angioendothelioma and granulomas like Wegener's granulomatosis and midline granuloma should be ruled out. Conversely when a bleeding necrotic mass is seen in the nose and paranasal sinuses of an adult male, a metastatic renal cell carcinoma should be suspected and the primary site, i.e. kidneys, should be evaluated as biopsy from the bleeding mass may be difficult and the necrotic tissue may be inconclusive on histopathology.

\section{REFERENCES}

1. Lim R.Y., Bastug D.F., Caldwell B.L. (1989): Metastatic renal cell carcinoma of the nasal septum. WV Med J 85: 143-145.

2. Matsumoto Y., Yanagilhara N. (1982): Renal cell carcinoma metastatic to the nose and paranasal sinuses. Laryngoscope 92: 1190-1193.

3. Som P.M., Norton K.I., Shugar J.M.A et al. (1987): Metastatic hypernephroma to the head and neck. Am J Neuroradiol 8: 11031106.

4. Cited by Wustrow F. (1965): Die Tumoren des Gesichtsschadels Topographie, Pathologie, und Klinik. Urban and Schwarzenberg, Munchen u. Berlin 195.

5. Boles R., Cerny J. (1971): Head and neck metastasis from renal carcinomas. Mich Med 70: 616-618.

6. Bernstein J.M., Montgomery W.W., Bologh K. (1966): Metastatic tumors to the maxilla, nose and paranasal sinuses. Laryngoscope 76: $621-650$.

7. Nason R., Carrau R.L. (2004): Metastatic renal cell carcinoma to 
the nasal cavity. American Journal of Otolaryngology 25(1): 54-57.

8. Nahum A.M., Bailey B.J. (1963): Malignant tumours metastatic to the paranasal sinuses. Case report and review of the literature. Laryngoscope 83: 942-953.

9. Gottlieb M.D., Roland J.T. (1998): Paradoxical spread of renal cell carcinoma to the head and neck. Laryngoscope 108(9): 13011305 .

10. Pantuck A.J., Zisman A., Belldegum A. (2001): Gene and immune therapy for renal cell carcinoma. Int J Urol 8: s1-4.

11. Johnson I.J., Campbell J.B. (1993): Renal derived epistaxis. J Laryngol Otol 107 144-145.

12. Lim L.H.Y., Goh C.H.K..(2001): A rare case of renal cell carcinoma metastatic to sinonasal area. Ear Nose Throat 80: 462-467.

13. Simo R., Sykes A.J., Hargreaves S.P. et al (2000); Metastatic renal cell carcinoma to the nose and paranasal sinuses. Head Neck 22: 722-727.
14. de Kernion J.B., Sarna G., Figlin R. et al.(1983): The treatment of renal cell carcinoma with human leukocyte alpha-interferon. J Urol 130: $1063-1066$.

15. Quesado J.R., Swanson D.A., Gutterman J.U. (1985): Phase II study of interferon alpha in metastatic renal-cell carcinoma: $A$ progress report. J Clin Oncol 3: 1086-1092.

Address for correspondence

Dr. Dipak Ranjan Nayak MS, FUICC, FICS

Professor \& Head of unit II,

Department of Otolaryngology - Head \& Neck Surgery,

Kasturba Medical College \& Hospital, Manipal 576104, Karnataka, India. email: drnent@rediffmail.com

Phone: 0820-2922143 (o)

Fax No: 0820-2570061

\title{
A RARE CASE OF INFLAMMATORY PSEUDOTUMOUR OF THE SUBMANDIBULAR LYMPHNODE.
}

\author{
Anjana Trivedi,* Jitendra Patel.**
}

\begin{abstract}
Inflammatory pseudotumour (IPT), also kiown as inflinmatory myofibroblastic tumour, is a rare cause of benign cervical lymphadenopathy. In its acute clinical presentation it mimics malignant and infective causes of cervical tymphadenopathy. The combined ctivical history, histopathological findings, and imaging features of an infitrating soft-tissue mass should lead to the possibility of iPT. Radical surgery should be avoided before there is histological proof of a malignant tumor.
\end{abstract}

Keywords: Inflammatory pseudotumour, submandibular lymphnode, radio-patho findings, steroids.

\section{INTRODUCTION}

Inflammatory pseudotumor (IPT) is a rarely occurring lesion. The condition was first described in 1905 by Birch-Hirschfield. Because an inflammatory pseudotumor has an abundance of polyclonal plasma cells, its etiology (postinflammatory vs immunologic) is still being debated. The term pseudotumor was coined because these lesions mimic expansive, invasive, malignant tumors, both clinically and radiologically. IPT most commonly involves the lung and orbit. It is rarely found in the head and neck outside the orbits. The condition may mimic a malignant lesion, and may occur at sites that hinder or preclude biopsy, possibly exposing the patient to the risk of unnecessary and potentially mutilating surgery. The combined clinical history, histopathological and radiological features of a soft-tissue mass can be helpful in the diagnosis of IPT. In confirmed cases of IPT, conservative steroid therapy may cure the disease.

\section{CASE REPORT}

An 20 year old female patient was admitted with a 5 months history of swelling of the left submandibular region. Clinically it was like a submandibular gland tumour. CT scan of neck showed a poorly defined, enhancing soft-tissue mass lying against the enlarged and enhancing left submandibular salivary gland, extending into the masticator space (Fig 1). An MR study confirmed the mass lesion in the right masticator space, which appeared slightly hyperintense relative to muscle on T2-weighted SE images, and clearly enhancing on postcontrast T1-weighted SE images. The differential diagnosis included neoplastic lesion of submandibular lymphnode with inflammation of the submandibular salivary gland. FNAC revealed reactive inflammatory tissue with fibrous tissue and inflammatory cells. A lymphoid infiltrate and multiple vascular structures were recognized. No malignant cells seen. Higher antibiotic (Tab. 